\title{
First-Principles Investigation of Ag-Doped Gold Nanoclusters
}

\section{Xiao-Dong Zhang ${ }^{1}{ }^{*}$, Mei-Li Guo ${ }^{2}$, Di Wu ${ }^{1}$, Pei-Xun Liu ${ }^{1}$, Yuan-Ming Sun ${ }^{1}$, Liang-An Zhang ${ }^{1}$, Yi She ${ }^{1}$, Qing-Fen Liu ${ }^{1}$ and Fei-Yue Fan ${ }^{1}$ *}

1 Tianjin Key Laboratory of Molecular Nuclear Medicine, Institute of Radiation Medicine, Chinese Academy of Medical Sciences and Peking Union Medical College, Tianjin 300192, China;

E-Mails: wudi521wan@163.com (D.W.); pharm8888@yahoo.com.cn (P.-X.L.); yuanmings1962@163.com (Y.-M.S.); zhangla43@yahoo.com.cn (L.-A.Z.); yi_she2005@yahoo.com.cn (Y.S.); qingfenliu@yahoo.com.cn (Q.-F.L.)

2 Department of Physics, Tianjin Institute of Urban Construction, Tianjin 300384, China; E-Mail: guomeili@tjuci.edu.cn

* Authors to whom correspondence should be addressed; E-Mails: xiaodongzhang@ yahoo.cn (X.-D.Z.); feiyfan@gmail.com (F.-Y.F.); Tel.: +86-22-8568-3173; Fax: +86-22-8568-3173.

Received: 18 February 2011; in revised form: 28 April 2011 / Accepted: 6 May 2011 /

Published: 9 May 2011

\begin{abstract}
Gold nanoclusters have the tunable optical absorption property, and are promising for cancer cell imaging, photothermal therapy and radiotherapy. First-principle is a very powerful tool for design of novel materials. In the present work, structural properties, band gap engineering and tunable optical properties of Ag-doped gold clusters have been calculated using density functional theory. The electronic structure of a stable $\mathrm{Au}_{20}$ cluster can be modulated by incorporating $\mathrm{Ag}$, and the HOMO-LUMO gap of $\mathrm{Au}_{20-n} \mathrm{Ag}_{n}$ clusters is modulated due to the incorporation of $\mathrm{Ag}$ electronic states in the HOMO and LUMO. Furthermore, the results of the imaginary part of the dielectric function indicate that the optical transition of gold clusters is concentration-dependent and the optical transition between HOMO and LUMO shifts to the low energy range as the Ag atom increases. These calculated results are helpful for the design of gold cluster-based biomaterials, and will be of interest in the fields of radiation medicine, biophysics and nanoscience.
\end{abstract}

Keywords: first-principles; gold clusters; electronic structure; optical properties 


\section{Introduction}

Gold nanostructures have attracted considerable attention owing to their unique electronic and optical properties, as well as their great potential for medical applications [1-3]. Today, gold nanostructures are also promising for their possible applications in photothermal therapy, radiotherapy and cancer cell imaging because of their good biocompatibility [4-6]. However, a disadvantage of photothermal therapy is that the optical absorption of gold nanoparticles, well known surface plasmon resonance (SPR), is always located in the visible light region, which limits the wider application of photothermal therapy in vivo. To utilize the high optical transmission of biotissue, it is necessary to enhance the near-infrared (NIR) optical absorption. In recent years, several groups have developed the gold nanorod, nanocage and core/shell structure, which are recognized as the promising materials for photothermal therapy $[1,7,8]$. However, the increasing size would obviously reduce the permeability of tumor tissue, which is disadvantageous to photothermal therapy.

Small gold clusters of typically 1-2 $\mathrm{nm}$ are proposed as alternative materials. Sun et al. have shown that the $\mathrm{Au}-\mathrm{SiO}_{2}$ clusters can induce the unusual optical transition, and the optical absorption can be modulated to the NIR. This is also confirmed by the recent photothermal therapy and drug delivery experiment [9]. However, the gold clusters are structurally unstable and have ambiguous electronic structure. The procedure of designing gold clusters with NIR optical absorption is influenced obviously, because of the long time controversy, by the structure of gold clusters. Recently, the obvious progressing has been achieved by density functional theory (DFT) theory calculations and optical spectra experiments $[10,11]$. The common view is that gold clusters prefer the two dimension structure in the range of $\mathrm{Au}_{4}-\mathrm{Au}_{13}$, while the $\mathrm{Au}_{14}-\mathrm{Au}_{20}$ show the cage-like three dimension structure [12]. The more complex cage-like $\mathrm{Au}_{32}-\mathrm{Au}_{38}$ clusters have been predicted, although the experimental result may always be contradicted. The optical absorption of $A_{2}-A_{13}, A u_{19}$, and $A_{2} u_{20}$ have been calculated by time-dependent density functional theory (TDDFT), while the optical transition of $\mathrm{Au}_{32}$ is also focused due to its more stable structure. Notably, the tetrahedral $\mathrm{Au}_{20}$ cluster shows a band gap of $\sim 1.818 \mathrm{eV}$, and shows slight NIR absorption [13,14].

Doping Au clusters by other metals provides an available route to modulate electronic and optical properties [15]. It has been demonstrated that metal atoms, such as $\mathrm{Zn}$ and $\mathrm{Cu}$, used to dope $\mathrm{Au}$ clusters can modify both their structural stability and optical properties due to the delocalizing $s$ and $d$ electronic configurations [16]. Bonacic-Koutecky et al. studied the structure and electronic properties of bimetallic $\operatorname{Ag}_{M} \mathrm{Au}_{N}(3<(\mathrm{M}+\mathrm{N})<5)$ clusters with DFT calculations [17]. For these clusters, the charge transferred from Ag to Au plays a dominant role in the structure of the bimetallic clusters. The $\mathrm{Au}-\mathrm{Ag}$ bond is preferred to the $\mathrm{Au}-\mathrm{Au}$ or $\mathrm{Ag}-\mathrm{Ag}$ bond [18]. In addition, the metal-doped $\mathrm{Au}$ clusters may modulate the HOMO-LUMO gap [19,20]. Thus, we are interested in whether AuAg cluster has tunable optical absorption properties.

Here, we studied electronic structure and optical properties of Ag-doped $\mathrm{Au}_{20}$ clusters. The paper is organized as follows. Section 2 presents and discusses the results of our calculations. First, we investigated the structural properties by analyzing the binding energy. Then we calculated the electronic structures, because the optical properties depend on both the interband and intraband transitions, which are determined by electronic states. Finally, we analyzed the optical transition in 
different configurations. Section 3 describes the basic ingredients and details of computational methods we applied. Section 4 concludes and summarizes our findings.

\section{Results and Discussion}

\subsection{Structural Properties of Ag-Doped Gold Clusters}

Figure 1 gives the calculated ground state geometries of $\mathrm{Au}_{20-n} \mathrm{Ag}_{n}$. The stability of gold nanostructure is very important for its electronic and optical properties. Thus, the binding energy per atom $E_{b}(n)$ of gold clusters is calculated to evaluate the stability of gold clusters, which are defined by the following formula:

$$
E_{b}(n)=\frac{E(\mathrm{Ag})+n E(\mathrm{Au})-n E\left(\mathrm{Au}_{n} \mathrm{Ag}\right)}{n+1}
$$

where $E(\mathrm{Ag}), E(\mathrm{Au})$, and $E\left(\mathrm{Au}_{n} \mathrm{Ag}\right)$ represent the total energies of the most stable $\mathrm{Ag}, \mathrm{Au}$, and $\mathrm{AuAg}$ clusters, respectively. It is worth pointing out that all of the clusters are found to prefer the lowest spin state. We find that the binding energy of $\mathrm{Au}_{20}$ is $2.40 \mathrm{eV}$, which is very close to the previous investigation of gold clusters [21]. It has been demonstrated that the binding energy increases with an increase in the size of gold clusters [11]. Increasing binding energy means increasing stability due to enhanced core electron configurations. After $\mathrm{Ag}$ doping, the binding energy of $\mathrm{Au}_{19} \mathrm{Ag}_{1}, \mathrm{Au}_{18} \mathrm{Ag}_{2}$, $\mathrm{Au}_{17} \mathrm{Ag}_{3}$, and $\mathrm{Au}_{16} \mathrm{Ag}_{4}$ are 2.68, 2.68, 2.68, and $2.69 \mathrm{eV}$, respectively. The increasing doping atom induces a tiny effect on the binding energy. It is worth noting that the binding energy of AuAg alloy is higher than the $\mathrm{Au}_{20}$. It shows that $\mathrm{Ag}$ atom incorporation can enhance the structural stability. Indeed, the $\mathrm{Au}-\mathrm{Ag}$ bond is stronger than the $\mathrm{Au}-\mathrm{Au}$ bond and gives an extra $\sigma$-bonding interaction by the overlap between the vacant $\mathrm{Ag} 4 p$ and valence $\mathrm{Au} 6 s(5 d)$ orbital, which is very similar to the previous Ag-doped gold clusters [20].

Figure 1. Calculated ground state geometries of $\mathrm{Au}_{20-} \mathrm{Ag}_{n}$.

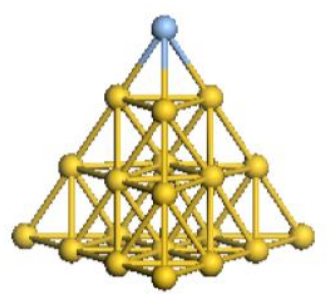

$$
\mathrm{Au}_{19} \mathrm{Ag}_{1}
$$

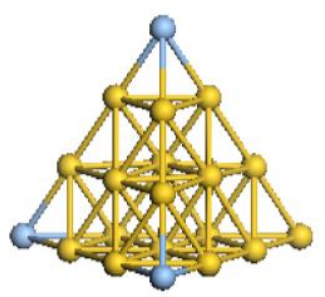

$$
\mathrm{Au}_{17} \mathrm{Ag}_{3}
$$

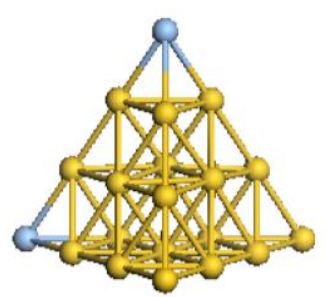

$\mathrm{Au}_{18} \mathrm{Ag}_{2}$

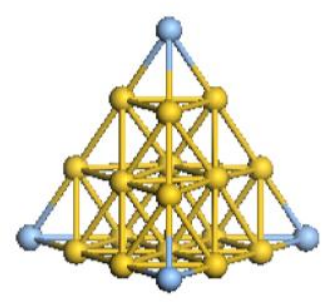

$\mathrm{Au}_{16} \mathrm{Ag}_{4}$ 


\subsection{Electronic Structure of Ag-Doped $A u_{20}$ Clusters}

Figure 2 shows the density of states (DOS) to reveal the electronic structure of $\mathrm{Au}_{20}{ }_{n} \mathrm{Ag}_{n}$ clusters. The $\mathrm{Au}_{20}$ cluster shows the large HOMO-LUMO gap, which is in good agreement with the other computational results [10,21]. The exact band gap of $\mathrm{Au}_{20}$ is $1.47 \mathrm{eV}$, which is less than the experimental data of $1.78 \mathrm{eV}$ (or $1.818 \mathrm{eV}$ ) due to the underestimation of electronic states by DFT [22]. Meanwhile, the $\mathrm{Au} d$ states are dominated in HOMO, and are located in the range of -6 and $0 \mathrm{eV}$. HOMO consists of $\mathrm{Au} s$ and $d$ states; the $\mathrm{Au} d$ states are dominant. It is clearly seen that when $n$ changes from 1 to 4 , the DOS also changes. In general, the band gap of $\mathrm{Au}_{20-}{ }_{n} \mathrm{Ag}_{n}$ clusters is less than that of the pure $\mathrm{Au}_{20}$ cluster expect for $\mathrm{Au}_{16} \mathrm{Ag}_{4}$. It confirms that the $\mathrm{Ag}$ incorporation into $\mathrm{Au}_{20}$ can induce the obvious effect on gap, which is consistent with the previous results $[19,23,24]$. The exact band gaps of $\mathrm{Au}_{20-} \mathrm{Ag}_{n}$ are 1.42, 1.34, 1.40, and $1.68 \mathrm{eV}$, which are corresponding to the different $n$ values from 1 to 4 . The variation of gap can be understood by electronic states. It can be seen that the LUMO of $\mathrm{Au}_{19} \mathrm{Ag}_{1}, \mathrm{Au}_{18} \mathrm{Ag}_{2}$, and $\mathrm{Au}_{17} \mathrm{Ag}_{3}$ is shifted to the low energy range compared with $\mathrm{Au}_{20}$, which can be clearly seen in Figure 2. This shift can lead to the obvious variation of electronic properties. As it is incorporated more and more, the $p$ states in LUMO become stronger, which leads to the LUMO shift and band gap narrowing. However, the LUMO of $\mathrm{Au}_{16} \mathrm{Ag}_{4}$ shift to high energy range, and thus induce the increase of the gap, which should be related to improving structural stability and enclosing electronic configurations [25]. Meanwhile, the $p$ and $s$ states are obviously enhanced in the LUMO, and these electronic states can have effects on optical properties.

Figure 2. The partial DOS of (a) $\mathrm{Au}_{20-} \mathrm{Ag}_{n}$ clusters.

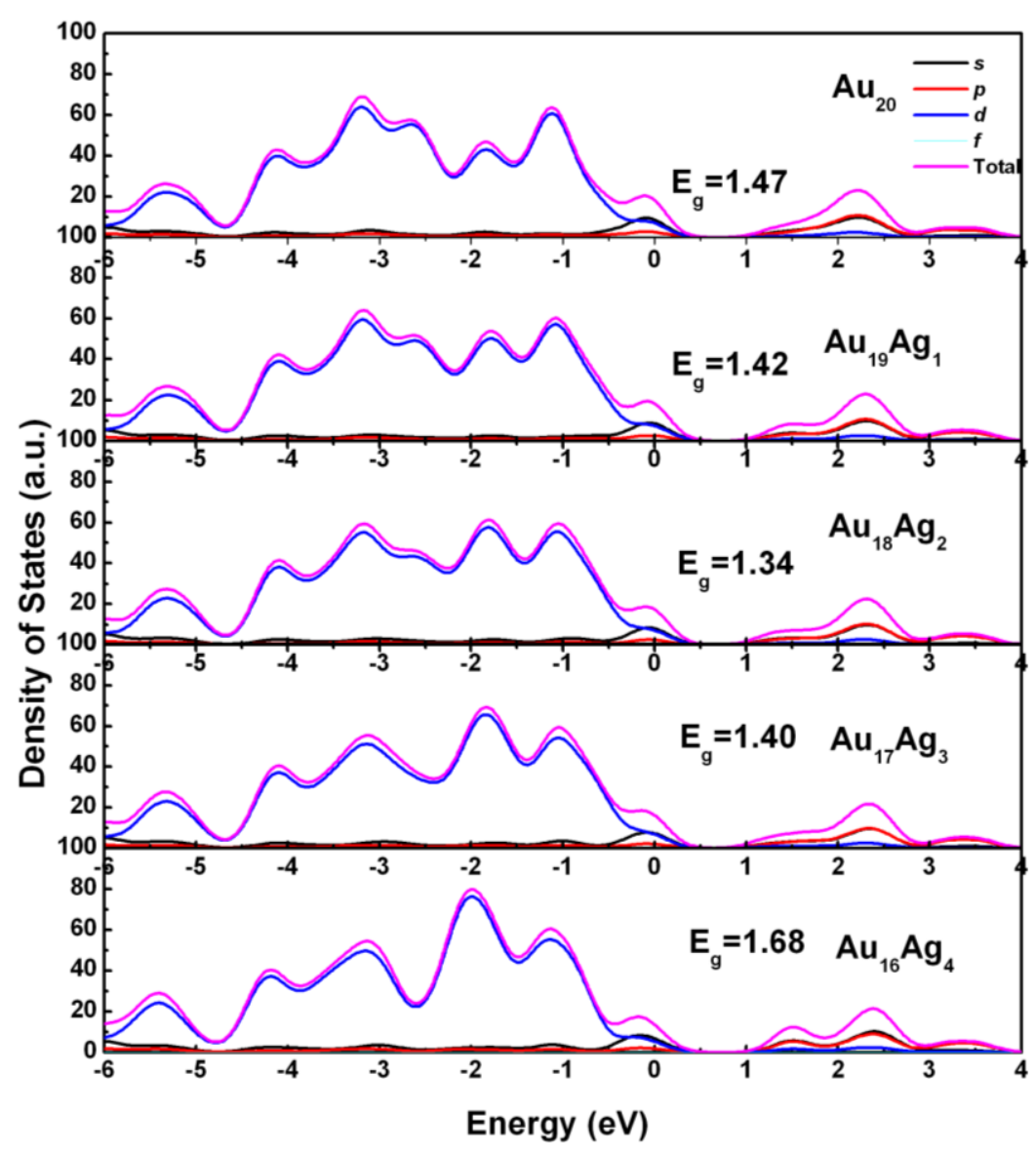




\subsection{Tunable Optical Properties of Gold Clusters}

For investigating the optical transition of $\mathrm{Au}_{20-} \mathrm{Ag}_{n}$ clusters, it is necessary to investigate the imaginary part of the dielectric function, because it is very important to the optical properties of any materials. Regarding $\mathrm{Au}_{20}$ in Figure.3, there are two main peaks in $\varepsilon_{2}(\omega)$, at 1.79 and $2.51 \mathrm{eV}$, respectively (namely $E_{1}, E_{2}$ ), which are very close to the previous results of 1.86 and 2.78 [21]. It can be seen in the inset of Figure 3 that direct transition can be caused between HOMO and LUMO. Therefore, it can be expected that $E_{1}(1.79 \mathrm{eV})$ should mainly be caused by optical transitions between $\mathrm{Au} 6 s 55 \%$ (and $\mathrm{Au} d, 45 \%$ ) states in HOMO and Au $6 s$ states in LUMO, which are close to the other first-principles evaluation $(1.86 \mathrm{eV})$ [21]. Moreover, it is not far from the HOMO-LUMO gap of $1.48 \mathrm{eV}$. In the $\mathrm{DOS}$ of $\mathrm{Au}_{20}$, the $\mathrm{Au} d$ states produce two peaks, 1.41 and $1.97 \mathrm{eV}$, which can induce some electronic states in the energy level range of $-4 \sim 0 \mathrm{eV}$. Thus, the optical transitions of $E_{2}$ can be due to the optical transitions between HOMO-1 consist of Au $d$ states and LUMO consist of Au $6 s$ (and $\mathrm{Au} p$ ) states.

Figure 3. The imaginary part of dielectric function $\varepsilon_{2}(\omega)$ of $\mathrm{Au}_{20}$ clusters.

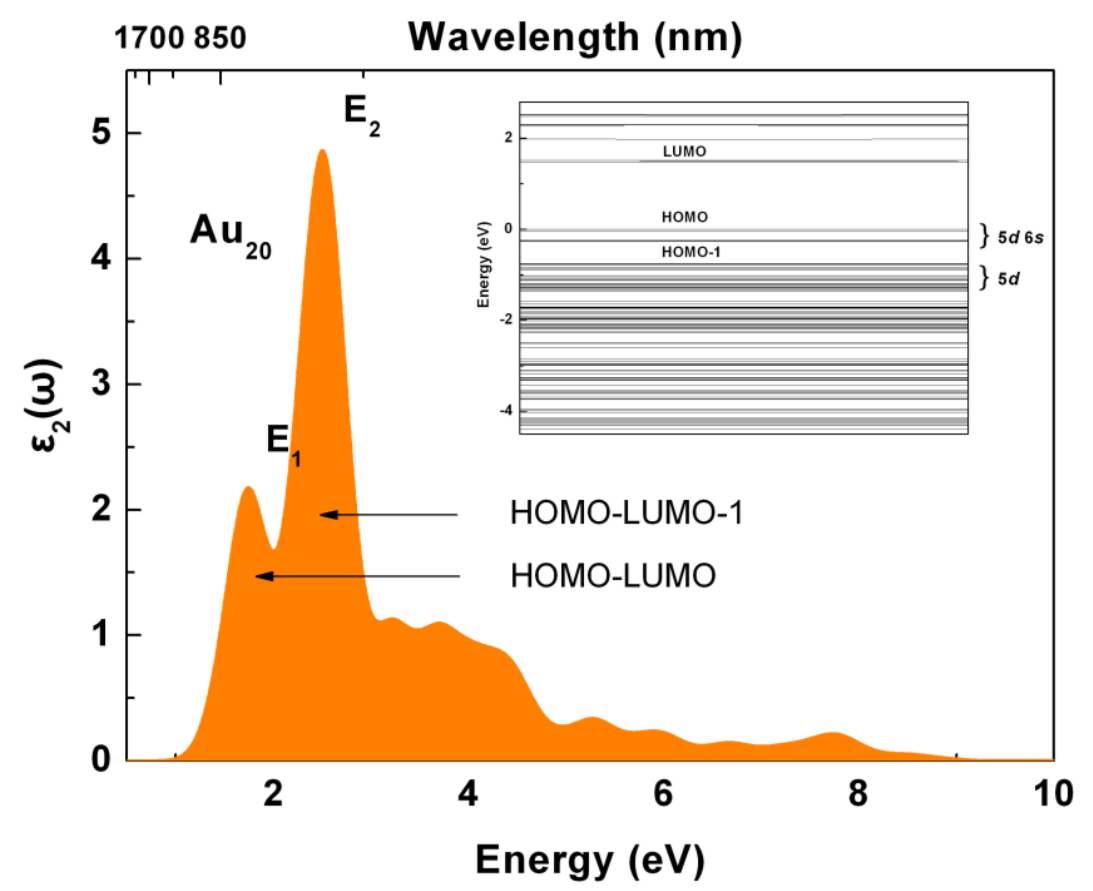

Figure 4 shows the imaginary part of dielectric function $\varepsilon_{2}(\omega)$ of $\mathrm{Au}_{20-} \mathrm{Ag}_{n}$ clusters. $\mathrm{Ag}$ incorporation induces some obvious variations of optical transitions. Firstly, $E_{1}$ has gradually disappeared, which can be related to the red-shift of $E_{2}$ and further inhibition of the intrinsic optical transition of $E_{1}$. Secondly, $E_{2}$ shows the tunable optical properties with the increasing $\mathrm{Ag}$ incorporation. The $E_{2}$ of $\mathrm{Au}_{20}, \mathrm{Au}_{19} \mathrm{Ag}_{1}, \mathrm{Au}_{18} \mathrm{Ag}_{2}, \mathrm{Au}_{17} \mathrm{Ag}_{3}$, and $\mathrm{Au}_{16} \mathrm{Ag}_{4}$ is 2.51, 2.35, 2.25, 2.07, and $2.01 \mathrm{eV}$, respectively. To understand these optical phenomena in detail, it is necessary to analyze the optical transition by electronic states. 
Figure 4. The tunable imaginary part of dielectric function $\varepsilon_{2}(\omega)$ of $\mathrm{Au}_{20-} \mathrm{Ag}_{n}$ clusters.

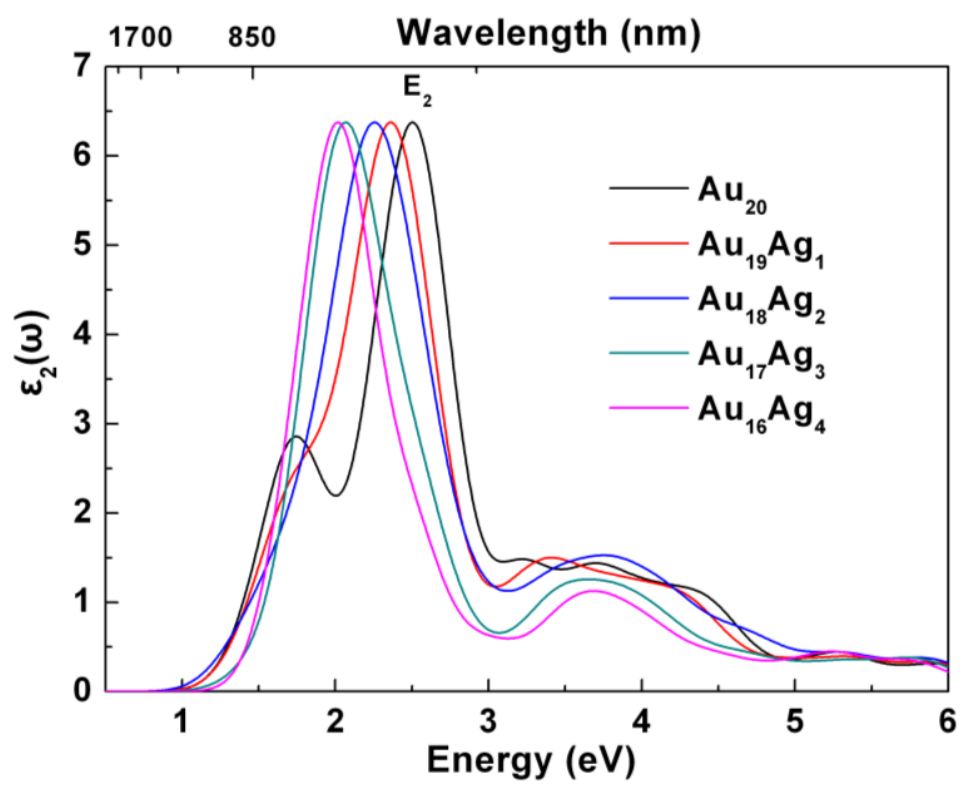

Figure 5 shows the transition energy level of $\mathrm{Au}_{20-} \mathrm{Ag}_{n}$ from band structure calculations. The optical transition between HOMO-LUMO has been affected by Ag incorporation. The variation in energy level can be described in two stages. In the first stage of $A_{19} A_{1}$ and $A_{18} A g_{2}, A g$ electronic states contribute to both HOMO and LUMO. Furthermore, LUMO has slightly shifted to the low energy range, which induces the decrease of transition level and can be responsible for the red-shifts of $\mathrm{E}_{2}$. In the second stage of $\mathrm{Au}_{17} \mathrm{Ag}_{3}$ and $\mathrm{Au}_{16} \mathrm{Ag}_{4}$, the increasing $\mathrm{Ag}$ atom induces more dispersive in $d$ states, and LUMO has shifted to the high energy range, which may cause gap variation. It has been supposed that the gap widening of $\mathrm{Au}_{16} \mathrm{Ag}_{4}$ is important evidence for the enhancement of binding energy and structural stability [25]. The heavy doping can have obvious effects on electronic states, and further investigation is still interesting. More valuable information is still necessary to probe by optical absorption.

Figure 5. The outline of optical transition of $\mathrm{Au}_{20-}{ }_{n} \mathrm{Ag}_{n}$ clusters calculated by energy level.

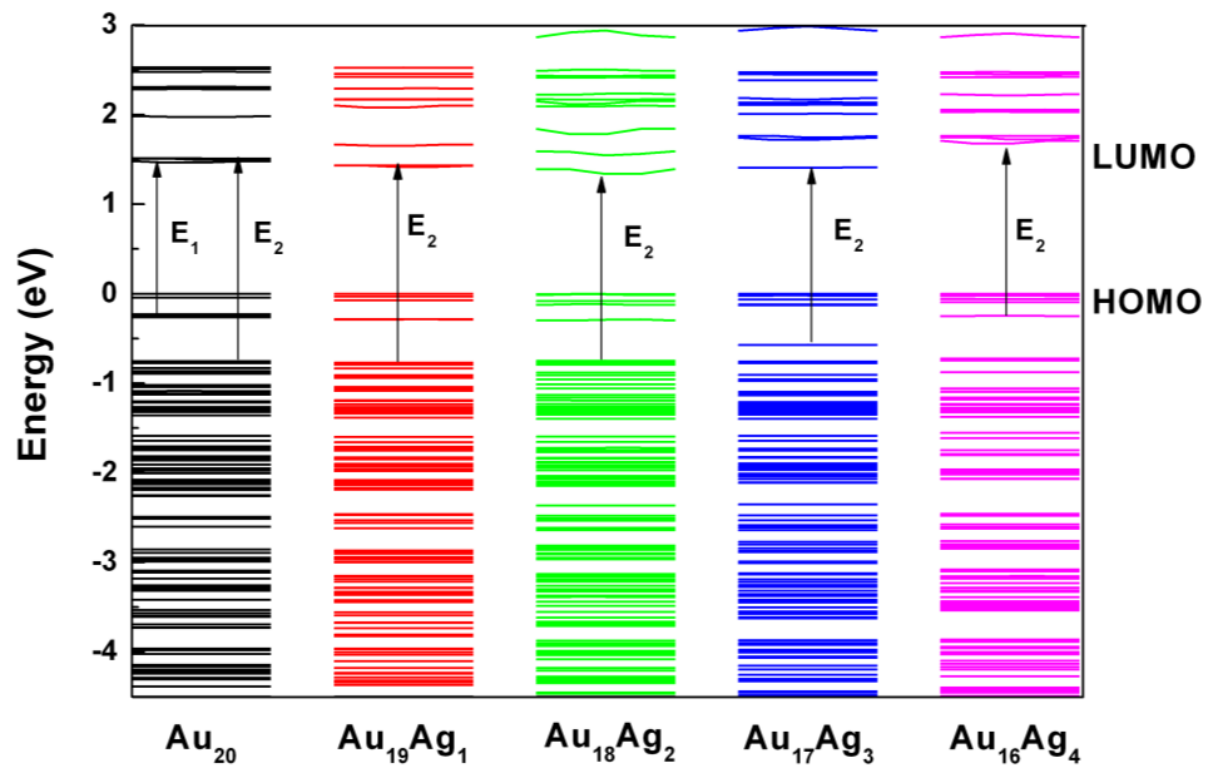


Figure 6 presents the optical absorption of $\mathrm{Au}_{20-} \mathrm{Ag}_{n}$ clusters. The dominant absorption around 450-550 nm can be observed in all $\mathrm{Au}_{20-} \mathrm{Ag}_{n}$ clusters. For $\mathrm{Au}_{20}$, the absorption band of $450 \mathrm{~nm}$ could be due to the optical transition of $E_{2}$, while the $707 \mathrm{~nm}$ absorption band is related to the intrinsic optical transition of $E_{1}$. The $\mathrm{Ag}$ incorporation induces the red-shift of absorption band $\left(E_{2}\right)$ from $478 \mathrm{~nm}\left(\mathrm{Au}_{19} \mathrm{Ag}_{1}\right)$ to $543 \mathrm{~nm}\left(\mathrm{Au}_{16} \mathrm{Ag}_{4}\right)$. We need to consider two possible effects on the optical absorption of these $\mathrm{Au}_{20-} \mathrm{Ag}_{n}$ clusters. On the one hand, the HOMO-LUMO gap can be underestimated by the DFT. According to the available optical data of $\mathrm{Au}_{20}$ from the experiment, the gap of tetrahedral $\mathrm{Au}_{20}$ is about $1.7-1.8 \mathrm{eV}$, which is larger $0.23-0.33 \mathrm{eV}$ than the calculated gap of $1.47 \mathrm{eV}$. Thus, the actual gap of $\mathrm{Au}_{20-} \mathrm{Ag}_{n}$ is wider than that of present calculated results. On the other hand, the structure stability of $\mathrm{Au}_{20-n} \mathrm{Ag}_{n}$ should also be taken into account. In the geometry calculations, the binding energy of $\mathrm{Au}_{16} \mathrm{Ag}_{4}$ is higher than that of the pure $\mathrm{Au}_{20}$ cluster and the other AuAg clusters, which indicates the doping feasibility. Therefore, more Ag atom incorporation may be promising for further fabrication, NIR absorption and related applications. Zorriasatein et al. have proposed that $\mathrm{Cu}$ incorporation into $\mathrm{Au}$ clusters can modulate the band gap effectively, and the results showed that the introduction of $\mathrm{Cu}$ enhanced the binding energy per atom compared to Au clusters [25]. AuAg clusters also showed good structural stability and optical properties [23,26,27]. Thus, it can be expected that $\mathrm{Ag}$ incorporation is an effective strategy for modulating optical properties of Au clusters. Our results also clearly show that Ag incorporation can modify structural stability and modulate the optical properties of $\mathrm{Au}_{20}$ clusters. These methods also have potential applications in understanding the optical properties of metal nanoclusters and designing materials for photothermal therapy.

Figure 6. Optical absorption of $\mathrm{Au}_{20-} \mathrm{Ag}_{n}$ clusters.

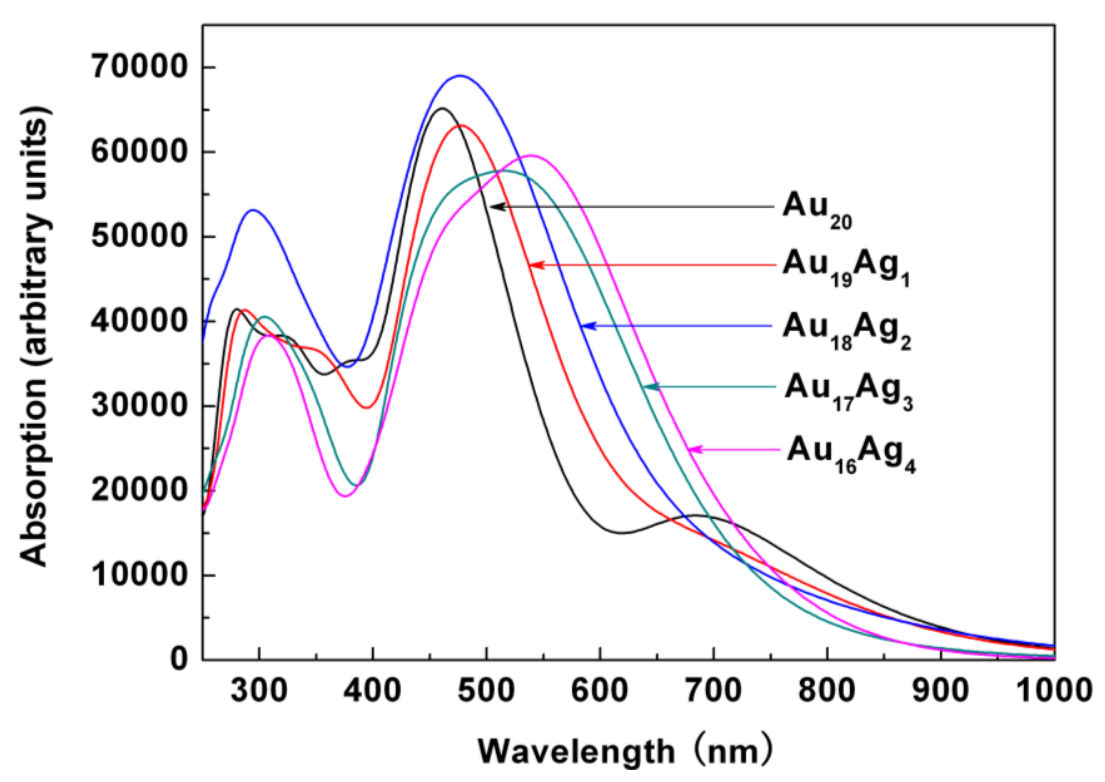

\section{Computational Section}

The calculations are based on density functional theory (DFT) using a plane-wave pseudopotential method [28]. We use the generalized gradient approximation (GGA) in the scheme of Perdew-Burke-Ernzerhof (PBE) to describe the exchange-correlation functional [29]. Norm-conserving 
pseudopotential is used to describe the electron-ion interaction. In this code, the plane wave functions of valence electrons are expanded in a plane wave basis set, and the use of norm-conserving pseudopotential allows a plane wave energy cutoff. Only plane waves with smaller kinetic energies are used in the expansion. Reciprocal-space integration over the Brillouin zone is approximated through careful sampling at a finite number of k-points using a Monkhorst-Pack mesh [30]. In the present system, full electron calculation for the $\mathrm{Au}_{20-} \mathrm{Ag}_{n}$ clusters is computationally rather expensive, so it is better to introduce effective core potentials for the $\mathrm{Au}$ and $\mathrm{Ag}$ atoms to describe the inner-core electrons. Under this approximation, the $5 d^{10} 6 s^{1}$ outermost valence electrons of the $\mathrm{Au}$ atom and $3 d^{10} 4 s^{2}$ outermost valence electrons of the Ag atom are described. It is well known that the interaction of a photon with the electrons in the system can be described in terms of time-dependent perturbations of the ground-state electronic states. Optical transitions between occupied and unoccupied states are caused by the electric field of the photon. The spectra from the excited states can be described as a joint density of states between the valence and conduction band. The momentum matrix elements, which are used to calculate the $\varepsilon_{2}(\omega)$, are calculated between occupied and unoccupied states which are given by the eigen vectors obtained as solution of the corresponding Schrödinger equation. Evaluating these matrix elements, one uses the corresponding eigen functions of each of the occupied and unoccupied states [22,28,31]:

$$
\varepsilon_{2}(\hbar \omega)=\left.\frac{2 e^{2} \pi}{\Omega \varepsilon_{0}} \sum_{k, v, c}\left|\psi_{k}^{c}\right| u \cdot r\left|\psi_{k}^{v}\right\rangle\right|^{2} \delta\left(E_{k}^{c}-E_{k}^{v}-\hbar \omega\right)
$$

where $\Omega$ is the volume of the elementary cell, $v$ and $c$ represent the valence and conduction bands, respectively, $k$ represents the $k$ point, $\omega$ is the frequency of the incident light, and $u$ is the vector defining the polarization of the electric field of the incident light, which is averaged over all spatial directions in the polycrystalline case. The real part of dielectric function $\varepsilon_{1}(\omega)$ can be evaluated from the imaginary part $\varepsilon_{2}(\omega)$ by the famous Kramer-Kronig relationship.

The calculations are performed at $20 \times 20 \AA$ supercell, which contains 20 neutral Au atoms. These models of gold clusters refer to the previous work, which has shown that the best structural stability and average distance between Au-Au bonding is about $2.73 \AA$ [21]. The substitutional method has been taken into account in this paper, and Ag atoms are used to substitute Au atoms in vertex site. In this way, the $\mathrm{Au}_{20-} \mathrm{Ag}_{n}$ clusters are built. In order to weaken the interaction with $\mathrm{Ag}-\mathrm{Ag}$, the separation distance should be as far as possible. We choose the energy cutoff to be $720 \mathrm{eV}$, and the Brillouin-zone sampling mesh parameters for the k-point set are $2 \times 2 \times 2$ for $\mathrm{Au}_{20}{ }_{n} \mathrm{Ag}_{n}$ clusters. The charge densities are converged to $2 \times 10^{-6} \mathrm{eV} /$ atom in the self-consistent calculation. And then, the $\mathrm{Au}_{20-n} \mathrm{Ag}_{n}$ clusters are optimized with lattice constants and the positions of substitutional atoms. In the optimization process, the energy change, maximum force, maximum stress and maximum displacement tolerances are set as $2 \times 10^{-5} \mathrm{eV} /$ atom, $0.05 \mathrm{eV} / \AA$, $0.1 \mathrm{GPa}$, and $0.002 \AA$, respectively.

\section{Conclusions}

In summary, a first-principles study has been performed to evaluate the electronic and optical properties of $\mathrm{Au}_{20-} \mathrm{Ag}_{n}$ clusters in different configurations. The $\mathrm{Au}_{20-n} \mathrm{Ag}_{n}$ clusters show better binding energy and structural stability than the $\mathrm{Au}_{20}$ cluster. The increasing $\mathrm{Ag}$ concentration can 
induce the HOMO-LUMO gap variation. Subsequently, the optical transition between HOMO-LUMO has shifted to the low energy range with the increasing Ag concentration. Tunable optical transition has been observed, and was shown to decrease from 2.51 to $2.01 \mathrm{eV}$ with the increase of Ag atoms. Our results clearly show that $\mathrm{Ag}$ incorporation can modulate the optical properties of $\mathrm{Au}_{20}$ clusters.

\section{Acknowledgements}

This work is supported by the National Natural Science Foundation of China (Grant No. 81000668), the Specialized Research Fund for Doctoral Program (SRFDP) of Higher Education State Education Ministry (Grant No. 200800231058), and the Subject Development Foundation of Institute of Radiation Medicine, CAMS (Grant No. SF1003).

\section{References}

1. Huang, X.; El-Sayed, I.; Qian, W.; El-Sayed, M. Cancer cell imaging and photothermal therapy in the near-infrared region by using gold nanorods. J. Am. Chem. Soc. 2006, 128, 2115-2020.

2. Hainfeld, J.; Slatkin, D.; Smilowitz, H. The use of gold nanoparticles to enhance radiotherapy in mice. Phys. Med. Biol. 2004, 49, N309-N315.

3. Wu, D.; Zhang, X.; Liu, P.; Zhang, L.; Fan, F.; Guo, M. Gold Nanostructure: Fabrication, Surface Modification, Targeting Imaging, and Enhanced Radiotherapy. Curr. Nanosci. 2011, 7, 110-118.

4. Murphy, C.; Gole, A.; Stone, J.; Sisco, P.; Alkilany, A.; Goldsmith, E.; Baxter, S. Gold nanoparticles in biology: beyond toxicity to cellular imaging. Acc. Chem. Res. 2008, 41, 1721-1730.

5. Zhang, X.; Wu, H. Toxicologic effects of gold nanoparticles in vivo by different administration routes. Int. J. Nanomed. 2010, 5, 771-781.

6. Zhang, X.D.; Guo, M.L.; Wu, H.Y.; Sun, Y.M.; Ding, Y.Q.; Feng, X.; Zhang, L.A. Irradiation stability and cytotoxicity of gold nanoparticles for radiotherapy. Int. J. Nanomed. 2009, 4, 165-173.

7. Loo, C.; Lowery, A.; Halas, N.; West, J.; Drezek, R. Immunotargeted nanoshells for integrated cancer imaging and therapy. Nano Lett. 2005, 5, 709-711.

8. Chen, J.; Wang, D.; Xi, J.; Au, L.; Siekkinen, A.; Warsen, A.; Li, Z.; Zhang, H.; Xia, Y.; Li, X. Immuno gold nanocages with tailored optical properties for targeted photothermal destruction of cancer cells. Nano. Lett. 2007, 7, 1318-1322.

9. Sun, Q.; Wang, Q.; Rao, B.; Jena, P. Electronic Structure and Bonding of Au on a $\mathrm{SiO}_{2} \mathrm{Cluster}^{\mathrm{A}}$ Nanobullet for Tumors. Phys. Rev. Lett. 2004, 93, 186803.

10. Gruene, P.; Rayner, D.; Redlich, B.; van der Meer, A.; Lyon, J.; Meijer, G.; Fielicke, A. Structures of neutral $\mathrm{Au}_{7}, \mathrm{Au}_{19}$, and $\mathrm{Au}_{20}$ clusters in the gas phase. Science 2008, 321, 674-676.

11. Li, X.; Wang, H.; Yang, X.; Zhu, Z.; Tang, Y. Size dependence of the structures and energetic and electronic properties of gold clusters. J. Chem. Phys. 2007, 126, 084505.

12. Zheng, X.; Shi, X.; Dai, Z.; Zeng, Z. Transport properties of the $\mathrm{Au}_{32}$ cluster with fullerene symmetry. Phys. Rev. B. 2006, 74, 85418.

13. Li, J.; Li, X.; Zhai, H.; Wang, L. Au 20 : A tetrahedral cluster. Science 2003, 299, 864-867.

14. Wu, K.; Li, J.; Lin, C. Remarkable second-order optical nonlinearity of nano-sized $\mathrm{Au}_{20}$ cluster: a TDDFT study. Chem. Phys. Lett. 2004, 388, 353-357. 
15. Guo, J.J.; Yang, J.X.; Die, D. First Principle Calculation on $\operatorname{Au}_{n} \mathrm{Ag}_{2}(n=1 \sim 4)$ Clusters. Commun. Theor. Phys. 2007, 48, 348-352.

16. Zhang, X.D.; Guo, M.L. Electronic structure and optical transitions of $\mathrm{Au}_{20-x} \mathrm{Cu}_{x}$ nanoclusters. J. Nanosci. Nanotechnol. 2010, 10, 7192-7195.

17. Bona čić-Koutecký, V.; Burda, J.; Mitri, R.; Ge, M.; Zampella, G.; Fantucci, P. Density functional study of structural and electronic properties of bimetallic silver-gold clusters: Comparison with pure gold and silver clusters. J. Chem. Phys. 2002, 117, 3120-3131.

18. Wang, L.; Pal, R.; Huang, W.; Zeng, X.; Wang, L. Observation of earlier two-to-three dimensional structural transition in gold cluster anions by isoelectronic substitution: $\mathrm{MAu}$ ( $n=8-11 ; \mathrm{M}=\mathrm{Ag}, \mathrm{Cu}$ ). J. Chem. Phys. 2010, 132, 114306.

19. Ghanty, T.; Banerjee, A.; Chakrabarti, A. Structures and the Electronic Properties of $\mathrm{Au}_{19} \mathrm{X}$ Clusters (X = Li, Na, K, Rb, Cs, Cu, and Ag). J. Phys. Chem. C 2009, 114, 20.

20. Li, S.; Shao, Z.; Han, S.; Xue, X.; Wang, F.; Sun, Q.; Jia, Y.; Guo, Z. Role of Ag-doping in small transition metal clusters from first-principles simulations. J. Chem. Phys. 2009, 131, 184301.

21. Idrobo, J.; Walkosz, W.; Yip, S.; Öğüt, S.; Wang, J.; Jellinek, J. Static polarizabilities and optical absorption spectra of gold clusters $\left(\mathrm{Au}_{n}, n=2-14\right.$ and 20) from first principles. Phys. Rev. $B$ 2007, 76, 205422.

22. Zhang, X.; Guo, M.; Li, W.; Liu, C. First-principles study of electronic and optical properties in wurtzite ZnCdO. J. Appl. Phys. 2008, 103, 063721.

23. Chen, F.; Johnston, R. Structure and spectral characteristics of the nanoalloy $\mathrm{Ag}_{3} \mathrm{Au}_{10}$. Appl. Phys. Lett. 2007, 90, 153123.

24. Lee, H.; Ge, M.; Sahu, B.; Tarakeshwar, P.; Kim, K. Geometrical and electronic structures of gold, silver, and gold-silver binary clusters: Origins of ductility of gold and gold-silver alloy formation. J. Phys. Chem. B 2003, 107, 9994-10005.

25. Zorriasatein, S.; Joshi, K.; Kanhere, D. Electronic and structural investigations of gold clusters doped with copper: $\mathrm{AuCu}(n=13-19)$. J. Chem. Phys. 2008, 128, 184314.

26. Rossi, G.; Ferrando, R.; Rapallo, A.; Fortunelli, A.; Curley, B.C.; Lloyd, L.D.; Johnston, R.L. Global optimization of bimetallic cluster structures. II. Size-matched $\mathrm{Ag}-\mathrm{Pd}, \mathrm{Ag}-\mathrm{Au}$, and $\mathrm{Pd}-\mathrm{Pt}$ systems. J. Chem. Phys. 2005, 122, 194309.

27. Curley, B.; Rossi, G.; Ferrando, R.; Johnston, R. Theoretical study of structure and segregation in 38-atom Ag-Au nanoalloys. Eur. Phys. J. D 2007, 43, 53-56.

28. Payne, M.; Teter, M.; Allan, D.; Arias, T.; Joannopoulos, J. Iterative minimization techniques for ab initio total-energy calculations: molecular dynamics and conjugate gradients. Rev. Mod. Phys. 1992, 64, 1045-1097.

29. Perdew, J.; Burke, K.; Ernzerhof, M. Generalized gradient approximation made simple. Phys. Rev. Lett. 1996, 77, 3865.

30. Monkhorst, H.; Pack, J. Special points for Brillouin-zone integrations. Phys. Rev. B 1976, 13, 5188.

31. Zhang, X.; Guo, M.; Liu, C.; Li, W.; Hong, X. Electronic and optical properties of TiCdO: A first-principles prediction. Appl. Phys. Lett. 2008, 93, 012103.

(C) 2011 by the authors; licensee MDPI, Basel, Switzerland. This article is an open access article distributed under the terms and conditions of the Creative Commons Attribution license (http://creativecommons.org/licenses/by/3.0/). 